\title{
Modification of Impulse Generation during Piqué Turns with Increased Rotational Demands
}

Antonia M. Zaferiou ${ }^{1}$, Rand R. Wilcox ${ }^{2}$, and Jill L. McNitt-Gray ${ }^{1,3}$

${ }^{1}$ Department of Biomedical Engineering, University of Southern California, Los Angeles, CA, USA;

${ }^{2}$ Department of Psychology, University of Southern California, Los Angeles, CA, USA;

${ }^{3}$ Department of Biological Sciences, University of Southern California, Los Angeles, CA, USA

Funding: This work was supported in part by USC Endowed Myronis Fellowship.

Corresponding Author: Antonia Zaferiou, email: zaferiou@alumni.usc.edu

Postdoctoral Research Fellow, Department of Mechanical Engineering, University of Michigan

\author{
Affiliation Address: \\ Jill McNitt-Gray \\ USC Biomechanics Research Laboratory \\ Departments of Biological Sciences and Biomedical Engineering \\ University of Southern California \\ 3616 Trousdale Parkway, AHF-252 \\ Los Angeles, CA 90089
}

Present Address:

Antonia Zaferiou

Department of Mechanical Engineering

University of Michigan

G.G. Brown Laboratory; 2350 Hayward St.

Ann Arbor, MI 48109-2125 


\begin{abstract}
During initiation of a piqué turn, a dancer generates impulse to achieve the desired lateral translation and whole-body rotation. The goal of this study was to determine how individuals regulate impulse generation when initiating piqué turns with increased rotational demands. Skilled dancers $(n=10)$ performed single $\left(\sim 360^{\circ}\right)$ and double $\left(\sim 720^{\circ}\right)$ piqué turns from a stationary position. Linear and angular impulse generated by the push and turn legs were quantified using ground reaction forces and compared across turn conditions as a group and within a dancer using probability-based statistical methods. The results indicate that as the rotation demands of the pique turn increased, the net angular impulse generated increased whereas net lateral impulse decreased. Early during turn initiation, the free moment contributed to angular impulse generation. Later during turn initiation, horizontal reaction forces were controlled to generate angular impulse. As rotational demands increased, the moment applied increased primarily from redirection of the horizontal reaction force (RFh) at the push leg and a combination of RFh magnitude and moment arm increases at the turn leg. RFh at each leg were coordinated to limit unwanted net linear impulse. Knowledge of observed subject-specific mechanisms is important to inform the design of turning performance training tools.
\end{abstract}

Keywords: Angular Impulse; Linear Impulse; Dance; Turn; Coordination; Biomechanics 


\section{Introduction}

Turning is a challenging and fundamental activity that is performed daily. While navigating through an environment, effective turning mechanics are needed to circumvent obstacles while maintaining balance and a functional pace. Turn performance reflects the ongoing interaction between the nervous system, musculoskeletal system, and reaction forces generated at the foot-surface interface (Flashner, Beuter, \& Arabyan, 1988). The trajectory of the center of mass (CM) is controlled relative to the base of support via reaction forces. The reaction force generation at each leg must be coordinated to ensure linear and angular momentum requirements of the task are satisfied while maintaining balance. These task requirements are potentially conflicting because generation of angular impulse about the $\mathrm{CM}$ involves reaction force components that are not aligned with the desired $\mathrm{CM}$ trajectory. For example, achieving the mechanical objectives during turning-while-walking can be particularly challenging for individuals with musculoskeletal and/or neural impairments (e.g., older adults at risk of falling, individuals with Parkinson's disease, stroke survivors, individuals with hip joint dysfunction, people using lower limb prostheses, etc.) (Hollands, Agnihotri, \& Tyson, 2014; Lam \& Luttmann, 2009; Segal, Orendurff, Czerniecki, Shofer, \& Klute, 2009; Spildooren et al., 2012; Tateuchi et al., 2014; Thigpen, Light, Creel, \& Flynn, 2000). Knowledge of how individuals satisfy multiple mechanical objectives under progressively more challenging conditions can assist personalization of intervention strategies to improve performance (McNitt-Gray et al., 2015).

Well-practiced turns performed by dancers provide a unique opportunity to understand how skilled individuals successfully satisfy multiple mechanical objectives. The piqué turn is a goal-directed task that imposes potentially conflicting mechanical objectives. The performance specifications for a piqué turn require that the $\mathrm{CM}$ translate only in the lateral direction while the body rotates about a vertical axis through the CM (Figure 1). Different amounts of whole-body rotation can be specified by the choreographer such that the rotational demands of the piqué turn can increase. Piqué turns are often initiated from a static position while the body mass is supported by the "push leg". During the "turn initiation phase", linear and angular impulse is generated, leading to the desired initial conditions of the "turn phase" that follows. During the turn phase, the CM continues to travel in the lateral direction and the body rotates while supported only by the "turn leg" (Figure 1).

There is a relatively small body of experimental research focused on how dancers initiate turns. Particularly, the initial stance configuration and subsystem momentum during the pirouette has been studied and is a typical focus of cueing during dance training (Kim, Wilson, \& Singhal, 2014; Lott \& Laws, 2012). However, training based on the initial stance configuration does not teach dancers how to interact with the ground to apply a moment throughout the turn initiation phase about a translating center of mass. During a piqué turn, applying a moment about a translating center of mass is particularly challenging because the desired translation is greater than it is during a pirouette and the turn is initiated with single leg support. During single-leg support phases of fouetté turns, study of the ground reaction forces revealed a large contribution of the free moment towards generating angular impulse at a time that the CM horizontal position is close to the center of pressure (Imura \& Yeadon, 2010). In the pique turn, the $\mathrm{CM}$ translates from its initial $\mathrm{CM}$ horizontal proximity to the center of pressure, which could provide an opportunity to use the horizontal reaction force (RFh) to apply a moment about the CM.

The piqué turn relates to pedestrian and athletic turns because the performer must satisfy translation and rotational goals during periods of single and double support. In the case of turning and cutting 
maneuvers, each leg plays a distinct role in generating the reaction forces required to control both linear and angular momentum of the body (Cinelli, Patla, \& Allard, 2008; Dixon et al., 2014; Hase et al., 1999; Jindrich, Besier, \& Lloyd, 2006; Jindrich \& Full, 1999). Identifying strategies expert turners use to manage multiple goals during multiple footfalls can have clinical implications. For example, dancers may use overarching strategies to initiate whole-body rotation during the piqué turn that exploit support leg(s) and $\mathrm{CM}$ advancement contexts found in pedestrian and athletic turns. The piqué turn initiation phase is most related to the "redirection" and "termination" footfalls in step turns and cutting maneuvers because linear and angular impulse are generated as the initial support leg externally rotates (Hase et al., 1999; Jindrich, Besier, \& Lloyd, 2006).The piqué turn initiation phase is also related to the "preparation" and "redirection" footfalls in spin turns because impulse is generated as support shifts to a leg that may externally rotate prior to ground contact and may spin during whole-body rotation (Cinelli, Patla, \& Allard, 2008; Dixon et al., 2014; Hase et al., 1999). In step and spin turns, older adults can fall as weight shifts between these footfalls because the moment about the center of mass in the vertical plane is too large to maintain the $\mathrm{CM}$ within the base of support during the initiation of wholebody rotation (Mathiyakom \& McNitt-Gray, 2008; Thigpen, Light, Creel, \& Flynn, 2000; Tinetti, Speechley, \& Ginter, 1988).

Studying the piqué turn also provides an opportunity to identify strategies used to accommodate increased rotational demands. Though subsystem momentum and initial stance configuration were previously studied in pirouettes with increased rotational demand (Imura \& Yeadon, 2010; Kim et al., 2014; Sugano \& Laws, 2002), the role of each leg towards linear and angular impulse generation has not been identified, despite its implications to help dance training. In a cutting maneuver with increased rotational demands, as moment of inertia about a vertical axis increased, the horizontal reaction force (RFh) did not change direction relative to the support leg and the RFh magnitude perpendicular to the $\mathrm{CM}$ advancement direction generally increased when the phase duration was similar (Qiao, Brown, \& Jindrich, 2014). Additionally, in the golf swing, increases in rotation requirements were satisfied by increasing the magnitude of the RFh at one or both legs (McNitt-Gray et al., 2013). In the case of the piqué turn performed with increased rotational demands, the RFh of each leg could increase, yet be coordinated so that the net linear impulse is aligned with the desired lateral trajectory of the CM.

The purpose of this study was to determine how individuals use both legs to regulate angular and linear impulse generated during the initiation phase of laterally translating turning tasks with increased rotational demands. We hypothesized that (1) as rotation demands of the turn increase, dancers will increase net angular impulse, (2) maintain net linear impulse generated, and (3) increase net angular impulse by increasing RFh magnitude at each leg. These hypotheses were tested by comparing how dancers generate linear and angular impulse while performing single and double piqué turns.

\section{Methods}

\subsection{Participants and Procedures}

Professional ballet and contemporary dancers with similar levels of experience and training ( $n=10$, female, $>9$ years of dance experience) volunteered to participate and provided informed consent in accordance with the institutional review board for human subjects. All subjects were free of lower extremity injury at the time of data collection. Prior to data collection, participants warmed up and practiced the experimental tasks until they felt familiar with the experimental setup. 
Dancers were asked to perform standard "en dedans" piqué turns that required rotation of the whole body while supported by one leg and lateral CM translation. Rotational demands were systematically increased by requiring varying degrees of whole-body rotation (single (pk1) $360^{\circ}$ rotation, double (pk2) $\sim 720^{\circ}$ rotation). The single and double piqué turns analyzed were started from a stationary position, and were followed by a single piqué turn along a lateral trajectory (consistent with typical ballet choreography). Task trajectory was identified using a target pole to remind the dancer of the desired direction of translation (along the $+Y$ lab axis, Figure 1). The duration of the turn's initiation phase was controlled using 80-82 bpm music, similar to timing restrictions in choreography dictated by tempo. Participants wore self-selected footwear and arranged for preferred frictional characteristics for turning (i.e., ballet slippers, rosin, etc.). Turns were performed until at least five successful turns were achieved under each condition. Five successful turns were typically achieved in less than seven attempts.

Reaction forces generated during each foot contact were measured using force plates $(1200 \mathrm{~Hz}$, Kistler, NY). Each forceplate and surrounding area was covered with dance flooring surface. The dance flooring selected provided typical frictional characteristics found on stages and in studios, but was rigid such that it did not interfere with force transmittance. Segment kinematics were captured using a retro-reflective 16-camera motion capture system $(100 \mathrm{~Hz}$, Natural Point Optitrak, OR) and Acquire3D software (CMotion, MD). These data were used to determine the initial CM position and to verify successful completion of tasks. The position of the $\mathrm{CM}$ was calculated by using segment parameters defined by de Leva (de Leva, 1996) and functional joint center calculations of the ankle, knee, and hip were used as segment endpoints (Schwartz \& Rozumalski, 2005). Horizontal CM trajectory was determined using the initial CM horizontal position of the turn initiation and the RFh generated by each leg (de Leva, 1996; Zatsiorsky \& King, 1998). RFh was normalized by body mass.

\subsection{Phase of Interest}

The pique turn performed in study was initiated from a static position with the body weight supported by the push leg (Figure 1). During the turn initiation phase, the linear and angular impulse needed to perform the pique turn is generated. The turn initiation phase was determined from the time when the lateral component of the RFh of the push leg exceeded $10 \mathrm{~N}$ until termination of push leg ground contact (Figure 1). Late in the turn initiation phase, there was a period of double-limb support after the initial contact of the turn leg with the ground (Figure 1, double support phase). Time zero was defined as the time that the push leg departed the forceplate at the end of the turn initiation phase (Figure 1). Therefore, data were aligned with time zero to compare across different trials, without time normalization.

\subsection{Data Analyses}

Differences in impulse generation between legs and turn conditions were determined by analyzing ground reaction forces. Angular impulse about a vertical axis passing through the $\mathrm{CM}$ during the turn initiation phase was determined using the discrete time integral of the moment. The moment applied about a vertical axis passing through the $\mathrm{CM}$ was determined by:

$$
\begin{aligned}
\text { Moment }_{c m} & =\left(\overrightarrow{r_{c m}} \times \overrightarrow{R F_{h}}\right)+T_{z} \\
& =\left(\left|\overrightarrow{r_{c m}}\right| \sin \theta\left|\overrightarrow{R F_{h}}\right|\right)+T_{z}
\end{aligned}
$$

where $\overrightarrow{r_{c m}}$ is the "position vector" (vector from the horizontal position of the center of mass to the point of horizontal reaction force application), $\overrightarrow{R F_{h}}$ is the horizontal reaction force, $T_{z}$ is the free moment (also known as "frictional torque" and "vertical torque") (Holden \& Cavanagh, 1991; International Society of Biomechanics, 2015), and $\theta$ is the angle between $\overrightarrow{r_{c m}}$ and $\overrightarrow{R F_{h}}$. To provide context for 
potential changes in position vector length, "stance size" was calculated as the distance between each leg's center of pressure when the turn leg made initial contact with the ground. These variables were calculated using custom MATLAB algorithms (Mathworks, Natick, MA).

To study the mechanisms used to increase the moment applied at each leg about the $\mathrm{CM}$, the variables contributing to the moment applied by each leg from the RFh were quantified during a subphase of each leg's ground contact time prior to peak moment applied by each leg. This subphase was defined as the $25 \%$ turn initiation phase duration prior to peak moment ("peak moment subphase") applied by each leg in each turn condition (boxed areas in Figure 5 show this subphase). This phase was selected to best represent what was modulated during the rise to peak moment applied by each leg for most dancers (Figure 5, boxed areas).

\subsection{Statistical Analyses}

Between task differences in mean angular impulse, lateral impulse, moment, and variables contributing to the moment applied about the CM under each turn condition (RFh magnitude, position vector length, $\sin \theta$, free moment, and stance size) were compared using statistical analysis software R (R Core Team, 2015). The probability that each variable was greater than or less than its value between turn conditions across dancers (i.e., as a group) were analyzed using a Sign Test. Within-subject comparisons of the probability for each variable of any single piqué trial being less than any double piqué trial was calculated. P-values were calculated for each dancer using Cliff's analog of the Wilcoxon-Mann-Whitney test (Cliff, 1996; Neuhäuser et al., 2007). This method was chosen because it performs well with small numbers of trials per condition (Neuhäuser et al., 2007). A step-down Fisher-type method was then applied to control the familywise error rate $(\alpha=0.05)$ over multiple comparisons, modified so that the level of significance becomes $\alpha / k$ at each $k^{\text {th }}$ iteration (Hochberg, 1988; Hochberg \& Tamhane, 1987; Wilcox \& Clark, 2015). The statistical method chosen provides more flexibility by allowing heteroscedasticity across dancers (Crowder \& Hand, 1990). This statistical method is highly dependent upon the distribution of $p$-values for each variable measured because the level for significance becomes $\alpha / k$ at each $k^{\text {th }}$ iteration (Wilcox $\&$ Clark, 2015). Therefore, the presentation of within-subject results provides a relatively conservative estimate of significant differences between turn conditions.

\section{Results}

\subsection{Angular Impulse Generation with Increased Rotation Demand}

Dancers generated greater mean net angular impulse during the initiation of double piqué turns compared to during single piqué turns (Figure 2). Significant differences in net angular impulse generated were observed as a group $(p=.001)$ and individually for four of 10 dancers (Figure 2$)$. The increase in mean net angular impulse from single to double piqué turns ranged from $8 \%$ to $119 \%$ within dancer. Eight of 10 dancers increased mean net angular impulse by more than $15 \%$.

\subsection{Linear Impulse Generation with Increased Rotation Demand}

As rotation demands of the turning task increased, the net lateral impulse generated during the initiation of piqué turns tended to decrease. The decrease in net lateral impulse generated was significant as a group $(p=.013$ ) and it was significant individually for two of 10 dancers (Figure 3 ). The decrease in lateral impulse generated by the push leg was significant as a group $(p=.013)$ and it was significant individually for three of 10 dancers (Figure 3). The difference in lateral impulse generated by the turn leg was not significant as a group $(p=.088)$ or individually (Figure 3 ). The mean change in lateral CM velocity during the turn initiation phase decreased by more than $0.05 \mathrm{~m} / \mathrm{s}$ when performing a double turn as compared to a single turn in eight of the 10 dancers (Figure 3 ). 
By coordinating the reaction force generation between legs, the net horizontal impulse in the anteroposterior direction remained minimal with increases in rotational demands (Figure 4). The magnitude of changes in $\mathrm{CM}$ velocity in the anteroposterior direction tended to be less than $0.2 \mathrm{~m} / \mathrm{s}$, which is consistent with the mechanical objectives of the piqué turn at the whole-body level. Withinsubject analyses indicated that as rotational demand increased, seven of 10 dancers increased the anterior impulse generated by the push leg and nine of 10 dancers increased the posterior impulse generated by the turn leg (Figure 4). However net impulse in the anterior posterior direction remained minimal, contributing to a change in anteroposterior velocity less than $0.2 \mathrm{~m} / \mathrm{s}$ for all turn conditions (Figure 4). As a group, the increase in anterior impulse generated by the push leg and turn leg were significant ( $p=.001$ push leg, $p=.001$ turn leg), though the net anteroposterior impulse was not significantly different $(p=.11)$.

\subsection{Reaction Force Regulation with Increased Rotation Demand}

As rotational demands increased, dancers increased the mean moment applied by one or both legs (exemplar Figure 5, Figure 6). Increases in mean moments at each leg were achieved by using subjectspecific modifications of reaction forces and moment arms (exemplar Figure 5, Figure 6). Significant differences in mean moment for each leg were observed as a group ( $p=.002$ push leg, $p=.001$ turn leg) and individually for two dancers (push leg, Figure 6A) and eight dancers (turn leg, Figure 6B). Dancers used subject-specific combinations of RFh regulation and position vector magnitude increases (Figure 6, exemplar Figure 5). Within-subject analyses indicated that the moment applied by the push leg increased primarily from reaction force redirection, without changes in the push leg's position vector length from the $\mathrm{CM}$ or increases in reaction force magnitude (Figure 6A). In contrast, the moment applied by the turn leg increased via a combination of reaction force redirection, increased reaction force magnitude, and increased position vector magnitude identified using within-subject analyses (Figure 6B). Despite differences in position vector magnitude at the turn leg with increased rotation, stance size did not increase or decrease significantly as a group or individually.

\subsection{Role of Each Leg towards Impulse Generation}

The majority of angular impulse was generated by the push leg for both turn conditions and all subjects (Figure 2, push leg vs. turn leg). Additionally, the majority of net lateral impulse generated during turn initiation for both single and double turns was generated by the push leg (Figure 3, push vs. turn leg). However, as rotational demand increased, the increase in angular impulse generated by the turn leg exceeded the increase in that generated by the push leg. Within-subject analysis indicated that eight of 10 dancers significantly increased mean angular impulse generated by the turn leg, while one dancer significantly increased mean angular impulse generated by the push leg (Figure 2).

\subsection{Temporal Contributions to Angular Impulse}

The net moment was generated by different mechanisms during the first half of the turn initiation phase compared to the second half of the turn initiation phase for both single and double piqué turns. Early in the turn initiation phase, before the turn leg initially contacted the ground, the free moment contributed to a majority of the push leg's angular impulse generation for all subjects and both turn conditions (Figure 5A). The free moment application was generally sustained for longer than half of the turn initiation phase, as in the exemplar data displayed (Figure 5A). Within-subject analyses indicated that two of 10 dancers increased the free moment significantly with increased rotation, which contributed to the observed increase in angular impulse generated by the push leg. Later in the turn initiation phase, typically after the turn leg made initial contact with the ground, the net moment 
applied increased to its peak magnitude during a relatively short period of time. Within-subject analyses indicated that six of 10 dancers initiated turn leg contact earlier within the turn initiation phase as rotation demand increased.

\section{Discussion}

This study identified differences in control strategies used by skilled dancers to regulate linear and angular impulse generation during well-practiced and goal-directed turns. Comparisons of reaction forces generated by each leg during the performance of single and double piqué turns performed by dancers provided an opportunity to determine how individuals successfully satisfy multiple mechanical objectives during turns. With increased rotation, net angular impulse generated increased but net linear impulse in the direction of travel decreased. Mechanisms used to increase net angular impulse generation between single and double piqué turns varied across dancers and between push and turn legs. For instance, Subject 2 and Subject 9 used opposite strategies to increase angular impulse generated by the push leg in single vs. double turns (Figure 7). Both dancers performed piqué turns in a similar fashion from a kinematics standpoint and generated similar angular impulse with their push leg for single and double turns (Figure 2). Both strategies successfully satisfy the mechanical objectives of the piqué turn, yet, are different from a musculoskeletal control perspective. For instance, a change in horizontal reaction force angle relative to the leg with increased rotation may require different muscle recruitment timing or muscle sets (Goh, Sullivan, Gordon, Wulf, \& Winstein, 2012; Macpherson, 1988a, 1988b; Ting \& Macpherson, 2005; Torres-Oviedo \& Ting, 2010), whereas, an increase in the horizontal reaction force magnitude may require scaling the muscle recruitment used during a single. Different mechanisms for regulating angular impulse generated by each leg (e.g. modify RFh direction and/or magnitude) are important to consider when designing interventions that aim to improve technique (J. L. McNitt-Gray et al., 2015). For example, Subject 2 and Subject 9 may benefit from different training feedback because they currently regulate RFh differently to successfully achieve an increase in angular impulse generation with increased rotational demands.

\subsection{Role of Each Leg to Increase Angular Impulse Generated}

During turns with increased rotational demands, the dancers tended to redirect the RFh generated by the push leg and used subject-specific combinations of increases in RFh magnitude and moment arm at the turn leg. Surprisingly, most dancers maintained or decreased the RFh magnitude at the push leg. These results are contrary to the RFh regulation strategy used during the golf swing, during which, increased rotation demands were satisfied by increasing the magnitude of the RFh of one or both legs (McNitt-Gray et al., 2013). However, the RFh redirection strategy may be advantageous in order to limit unwanted linear impulse while increasing $\sin \theta$ to generate more angular impulse.

The extent to which net angular impulse increased in double turns from additional angular impulse generated by the turn leg was surprising, considering the duration and kinematic context of the turn leg's ground contact. Specifically, the turn leg made contact with the ground using only the forefoot as a support surface during approximately $0.2 \mathrm{~s}$. The turn leg RFh magnitude was small (ranging from 0-30\% bodyweight), which facilitated balance on the turn leg during the turn phase immediately following. However, the mean moment applied by the turn leg during its rise to peak moment was similar to that applied by the turn leg (Figure 6). Upon turn leg ground contact, dancers pushed anteriorly on the ground such that the RFh contributed to impulse in the posterior direction. The portion of this phase with posteriorly-directed RFh tended to increase the moment applied by the turn leg and counter the 
anterior impulse generated by the push leg (that would otherwise translate the dancer anteriorly). In turning and cutting maneuvers with increased rotational demands, there may be a practical advantage to simplify neuromuscular control by amplifying the angular impulse generated during one footfall rather than increasing angular impulse generated by both footfalls. Additionally, this method of redirecting the RFh when its magnitude is relatively small (turn leg vs. push leg) may enable large increases to the moment applied about the center of mass without having large detriments to the linear impulse generation. In turning maneuvers outside of dance with increased rotational demand, this could mean that the "redirection" footfall of a spin turn or "termination" footfall of a step turn could become effective angular impulse regulators with little risk of disrupting the CM trajectory.

In order to increase the angular impulse generated by the turn leg, many dancers used an increase in turn leg ground contact time and/or an increase of magnitude of the position vector between the turn leg's point of force application and the body's CM. The increase of the turn leg's position vector magnitude tended to originate from a decrease in lateral $\mathrm{CM}$ velocity rather than a larger stance size. This is similar to the control strategies observed to facilitate balance in challenging gait tasks: increasing double-limb support duration and decreasing the CM speed while maintaining small-magnitude RFh (Dite \& Temple, 2002; Thigpen et al., 2000).

\subsection{Center of Mass Advancement context for Angular Impulse Generation}

During the first half of the turn initiation phase, before the turn leg contacted the ground, the free moment contributed to the majority of moment applied by the push leg. During this interval, the RFh at the push leg was directed towards the desired direction of travel. The relatively large free moment was sustained over half the duration of the turn initiation phase and attenuated when the turn leg made contact with the ground. The free moments measured in this study are of comparable magnitude to those previously reported for turning while walking (Dixon et al., 2014) and fouetté turns (Imura \& Yeadon, 2010). Further, using the phase of single-leg support early in turn initiation to generate the majority of the linear impulse was similar to findings of linear impulse generation during the foot contacts of the discus-throw (Yu et al., 2002). Generating the majority of the desired linear impulse prior to redirecting the RFh relative to the $\mathrm{CM}$ allowed dancers to take advantage of a larger position vector resulting from the $\mathrm{CM}$ translation away from its initial position above the push leg. These findings suggest that similar strategies could be used during turning while walking and cutting maneuvers: (1) use single-limb support to generate linear impulse and relatively large free moment, then (2) use double-limb support (and the advantage of a larger position vector as the $\mathrm{CM}$ advances) to redirect the reaction forces to generate more angular impulse, while simultaneously coordinating each leg to limit unwanted linear impulse.

\subsection{Limitations and Implications}

The experimental conditions in this study were designed to mimic dance studio conditions and elucidate an individual's mechanical strategies. Dancers participating in this study were able to establish their preferred foot-surface frictional characteristics by using their own preferred footwear. Therefore, the effect of footwear and dancer-preferred frictional settings was not established in this study. Additionally, music with a controlled tempo was used to standardize the speed of the turn initiation phase across participants, consistent with what a dancer is likely to encounter when satisfying constraints imposed by choreography. Though dancers did not increase turn initiation duration significantly with increased rotation, it is unknown if dancers would have increased the duration of the turn initiation phase with increased rotation without the tempo constraint imposed in this study. The 
within-dancer design provided an opportunity to determine how an individual dancer modified her own control strategies when performing turns with increased rotational demands (each individual served as her own control). Knowledge of these technique-related findings can assist in the design of customized feedback technologies to facilitate skill acquisition and improve dance performance (McNitt-Gray et al., 2015).

Overarching strategies used during this translating dance turn can be used to further understand strategies used during pedestrian and athletic turns. Dancers generated the majority of linear impulse during single-leg support (while generating angular impulse with the free moment) and used doublelimb support (and the advantage of a larger distance from the original ground contact) to redirect the reaction forces and generate the majority of the angular impulse. With increased rotational demands, dancers tended to increase the duration of double limb support, which allowed more time for redirecting the reaction forces, while limiting unwanted linear impulse through coordination between legs.

\section{Acknowledgements:}

The authors thank the dancers who participated in this study. Additionally, the authors thank the USC Biomechanics Research Lab, in particular, Travis Peterson and the undergraduate research assistants for their help during data collection. This work was supported in part by USC Endowed Myronis Fellowship. 


\section{References}

Cinelli, M. E., Patla, A. E., \& Allard, F. (2008). Strategies used to walk through a moving aperture. Gait \& Posture, 27(4), 595-602.

Cliff, N. (1996). Ordinal Methods for Behavioral Data Analysis. Mahwah, NJ: Lawrence Erlbaum Associates, Inc., Publishers.

Crowder, M. J., \& Hand, D. J. (1990). Analysis of Repeated Measures (1st ed.). New York: Chapman \& Hall/CRC.

Dite, W., \& Temple, V. a. (2002). A clinical test of stepping and change of direction to identify multiple falling older adults. Archives of Physical Medicine and Rehabilitation, 83(11), 1566-1571.

Dixon, P. C., Stebbins, J., Theologis, T., \& Zavatsky, A. B. (2014). Ground reaction forces and lower-limb joint kinetics of turning gait in typically developing children. Journal of Biomechanics, 45(15), 3726-33.

Flashner, H., Beuter, A., \& Arabyan, A. (1988). Fitting mathematical functions to joint kinematics during stepping: implications for motor control. Biological Cybernetics, 58, 9199.

Goh, H.-T., Sullivan, K. J., Gordon, J., Wulf, G., \& Winstein, C. J. (2012). Dual-task practice enhances motor learning: a preliminary investigation. Experimental Brain Research, 222(3), 201-10.

Hase, K., Stein, R. B., Chen, I., Yang, Y., Chan, R., \& Wang, R. (1999). Turning Strategies During Human Walking. Journal of Neurophysiology, 81, 2914-2922.

Hochberg, Y. (1988). A sharper Bonferroni test for multiple tests of significance. Biometrika, 75, 800-802.

Hochberg, Y., \& Tamhane, A. C. (1987). Multiple Comparison Procedures. Hoboken, NJ, USA: John Wiley \& Sons, Inc.

Holden, J. P., \& Cavanagh, P. R. (1991). The free moment of ground reaction in distance running and its changes with pronation. Journal of Biomechanics, 24(10), 887-97.

Hollands, K. L., Agnihotri, D., \& Tyson, S. F. (2014). Effects of dual task on turning ability in stroke survivors and older adults. Gait \& Posture, 40(4), 564-9.

Imura, A., \& Yeadon, M. R. (2010). Mechanics of the Fouetté turn. Human Movement Science, 29, 947-955.

International Society of Biomechanics. (2015). Kistler Force Plate Formulae. Retrieved from http://isbweb.org/software/movanal/vaughan/kistler.pdf

Jindrich, D. L., Besier, T. F., \& Lloyd, D. G. (2006). A hypothesis for the function of braking forces during running turns. Journal of Biomechanics, 39(9), 1611-20. 
Jindrich, D. L., \& Full, R. J. (1999). Many-Legged Maneuverability: Dynamics of Turning in Hexapods. The Journal of Experimental Biology, 202, 1603-1623.

Kim, J., Wilson, M., \& Singhal, K. (2014). Generation of vertical angular momentum in single, double, and triple-turn pirouette en dehors in ballet. Sports Biomechanics, 13(3), 215-229.

Lam, T., \& Luttmann, K. (2009). Turning capacity in ambulatory individuals poststroke. American Journal of Physical Medicine \& Rehabilitation / Association of Academic Physiatrists, 88(11), 873-83; quiz 884-6, 946.

Leva, P. De. (1996). Adjustments to Zatsiorsky-Seluyanov’s segment inertia parameters. Journal of Biomechanics, 29(9), 1223-1230.

Lott, M., \& Laws, K. (2012). The Physics of Toppling and Regaining Balance during a Pirouette. Journal of Dance Medicine \& Science, 16(4), 167-174.

Macpherson, J. M. (1988a). Strategies that simplify the control of quadrupedal stance. I. Forces at the Ground. Journal of Neurophysiology, 60(1), 204-217.

Macpherson, J. M. (1988b). Strategies that simplify the control of quadrupedal stance. II. Electromyographic activity. Journal of Neurophysiology, 60(1), 218-231.

Mathiyakom, W., \& McNitt-Gray, J. L. (2008). Regulation of angular impulse during fall recovery. Journal of Rehabilitation Research and Development, 45(8), 1237-48.

McNitt-Gray, J. L., Munaretto, J., Zaferiou, A., Requejo, P. S., \& Flashner, H. (2013). Regulation of reaction forces during the golf swing. Sports Biomechanics, 12(2), 121-31.

McNitt-Gray, J. L., Sand, K., Ramos, C., Peterson, T., Held, L., \& Brown, K. (2015). Using technology and engineering to facilitate skill acquisition and improvements in performance. Proceedings of the Institution of Mechanical Engineers, Part P: Journal of Sports Engineering and Technology, 229(2), 103-115.

Neuhäuser, M., Lösch, C., \& Jöckel, K.-H. (2007). The Chen-Luo test in case of heteroscedasticity. Computational Statistics \& Data Analysis, 51(10), 5055-5060.

Qiao, M., Brown, B., \& Jindrich, D. L. (2014). Compensations for increased rotational inertia during human cutting turns. Journal of Experimental Biology, 217(3), 432-443.

R Core Team. (2015). R: A Language and Environment for Statistical Computing. Vienna, Austria. Retrieved from https://www.r-project.org/

Schwartz, M. H., \& Rozumalski, A. (2005). A new method for estimating joint parameters from motion data. Journal of Biomechanics, 38(1), 107-16.

Segal, A., Orendurff, M., Czerniecki, J., Shofer, J., \& Klute, G. (2009). Transtibial amputee joint rotation moments during straight-line walking and a common turning task with and without a torsion adapter. Journal of Rehabil Research \& Development, 46(3), 375-384. 
Spildooren, J., Vercruysse, S., Meyns, P., Vandenbossche, J., Heremans, E., Desloovere, K., ... Nieuwboer, A. (2012). Turning and unilateral cueing in Parkinson's disease patients with and without freezing of gait. Neuroscience, 207, 298-306.

Sugano, A., \& Laws, K. (2002). Physical analysis as a foundation for pirouette training. Medical Problems of Performing Artists, 18(March), 29-32.

Tateuchi, H., Tsukagoshi, R., Fukumoto, Y., Akiyama, H., So, K., Kuroda, Y., \& Ichihashi, N. (2014). Compensatory turning strategies while walking in patients with hip osteoarthritis. Gait \& Posture, 39(4), 1133-7.

Thigpen, M. T., Light, K. E., Creel, G. L., \& Flynn, S. M. (2000). Turning Difficulty Characteristics of Adults Aged 65 Years or Older. Physical Therapy, 80(12), 1174-1187.

Tinetti, M., Speechley, M., \& Ginter, S. (1988). Risk factors for falls among elderly persons living in the community. New England Journal of Medicine, 319(26), 1701-1706.

Ting, L. H., \& Macpherson, J. M. (2005). A limited set of muscle synergies for force control during a postural task. Journal of Neurophysiology, 93(1), 609-13.

Torres-Oviedo, G., \& Ting, L. H. (2010). Subject-Specific Muscle Synergies in Human Balance Control Are Consistent Across Different Biomechanical Contexts. Journal of Neurophysiology, 103(6), 3084-3098.

Wilcox, R., \& Clark, F. (2015). Robust Multiple Comparisons Based on Combined Probabilities From Independent Tests. Journal of Data Science, 13(1), 1-11.

Yu, B., Broker, J., \& Silvester, L. J. (2002). A kinetic analysis of discus-throwing techniques. Sports Biomechanics, 1(1), 25-46.

Zatsiorsky, V. M., \& King, D. L. (1998). An algorithm for determining gravity line location from posturographic recordings. Journal of Biomechanics, 31(2), 161-164. 


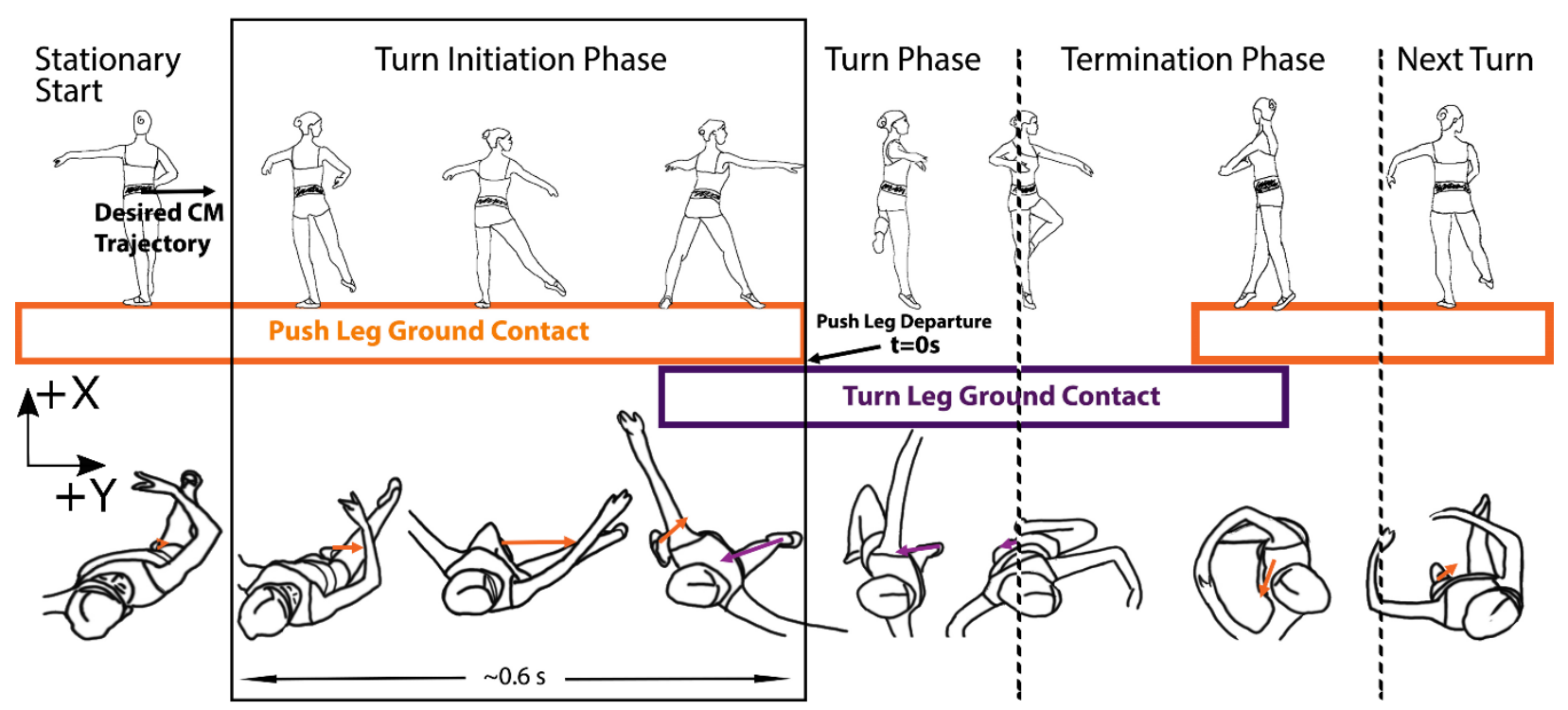

Figure 1: Phases of the piqué turn with exemplary kinematics and resultant horizontal reaction forces (overhead-view arrows) for a single piqué turn (Subject 1). During the turn initiation phase, the linear and angular impulse needed to perform the piqué turn is generated. The objective of the piqué turn is to translate laterally (along the $+Y$ lab axis) and complete rotations about a vertical axis through the center of mass (CM). The turn initiation phase was determined from the time when the $+Y$ component of the resultant horizontal reaction force of the push leg exceeded $10 \mathrm{~N}$ until termination of push leg ground contact. Late in the turn initiation phase, there is a short period of double-limb support after the initial contact of the turn leg with the ground. 


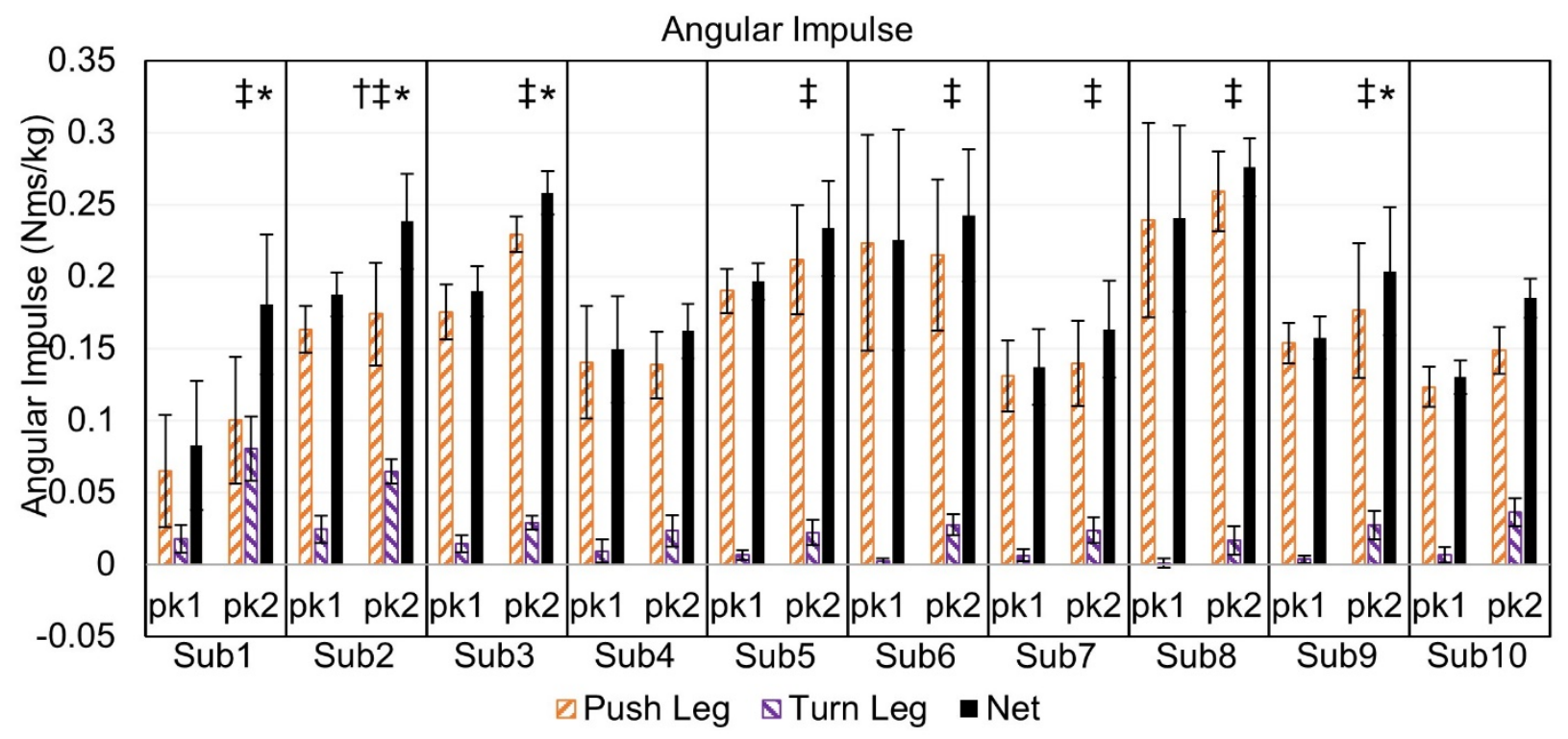

Figure 2: Mean (SD) normalized angular impulse generated by the push leg and turn leg of each dancer (Sub 1-10) during the turn initiation phase of single (pk1) and double (pk2) piqué turns. Symbols indicate within-subject significant differences in impulse generated by the push leg $(\dagger)$, turn leg $(\ddagger)$, and net impulse generated $(*)$ between turn conditions when tested at $\alpha=.05$ level and adjusted for multiple comparisons. The mean net angular impulse (black) increased as the rotation requirements increased ( $p$ $=0.001$ ). The push leg dominated the angular impulse generation during the turn initiation phase of both the single and double turns. As a group, angular impulse generated by each leg was significantly greater in doubles than in singles ( $p=.013$ push leg, $p=.002$ turn leg). 




Figure 3: Mean (SD) normalized linear impulse generated in the lateral direction by the push leg and turn leg of each dancer (Sub 1-10) during the turn initiation phase of single (pk1) and double (pk2) piqué turns. Symbols indicate within-subject significant differences in impulse generated by the push leg $\left(^{\dagger}\right)$, turn leg $(\ddagger)$, and net impulse generated $\left({ }^{*}\right)$ between turn conditions when tested at $\alpha=.05$ level and adjusted for multiple comparisons. Net lateral linear impulse generated (black) tended to decrease with increased rotational demand, however, not for all dancers. The decrease in net lateral impulse generated was significant as a group $(p=.013)$, the decrease in lateral impulse generated by the push leg was significant as a group $(p=.013)$, and difference in lateral impulse generated by the turn leg was not significant as a group ( $p=.088$ ). The push leg (orange) dominated the lateral impulse generation of both single and double turns. 




Figure 4: Mean (SD) normalized anteroposterior impulse by the push leg and turn leg of each dancer (Sub 1-10) during the turn initiation phase of single (pk1) and double (pk2) piqué turns. Symbols indicate within-subject significant differences in impulse generated by the push leg $(\dagger)$, turn leg $(\ddagger)$, and net impulse generated $(*)$ between turn conditions when tested at $\alpha=.05$ level and adjusted for multiple comparisons. By coordinating reaction force generation between legs, the net horizontal impulse (black) in the anterior/posterior direction remained minimal with increases in rotational demands. Withinsubject analyses indicated that as rotational demand increased, seven of 10 dancers increased the anterior impulse generated by the push leg and nine of 10 dancers increased the posterior impulse generated by the turn leg. Changes in net anteroposterior impulse were not found to be significant as a group $(p=.11)$ though within-subject analysis detected that Subject 3 significantly decreased net anteroposterior impulse. Functionally, the net anteroposterior impulses generated were very small, which is consistent with the mechanical objectives of the piqué turn. 


\section{Exemplar Strategy to Increase Moment}

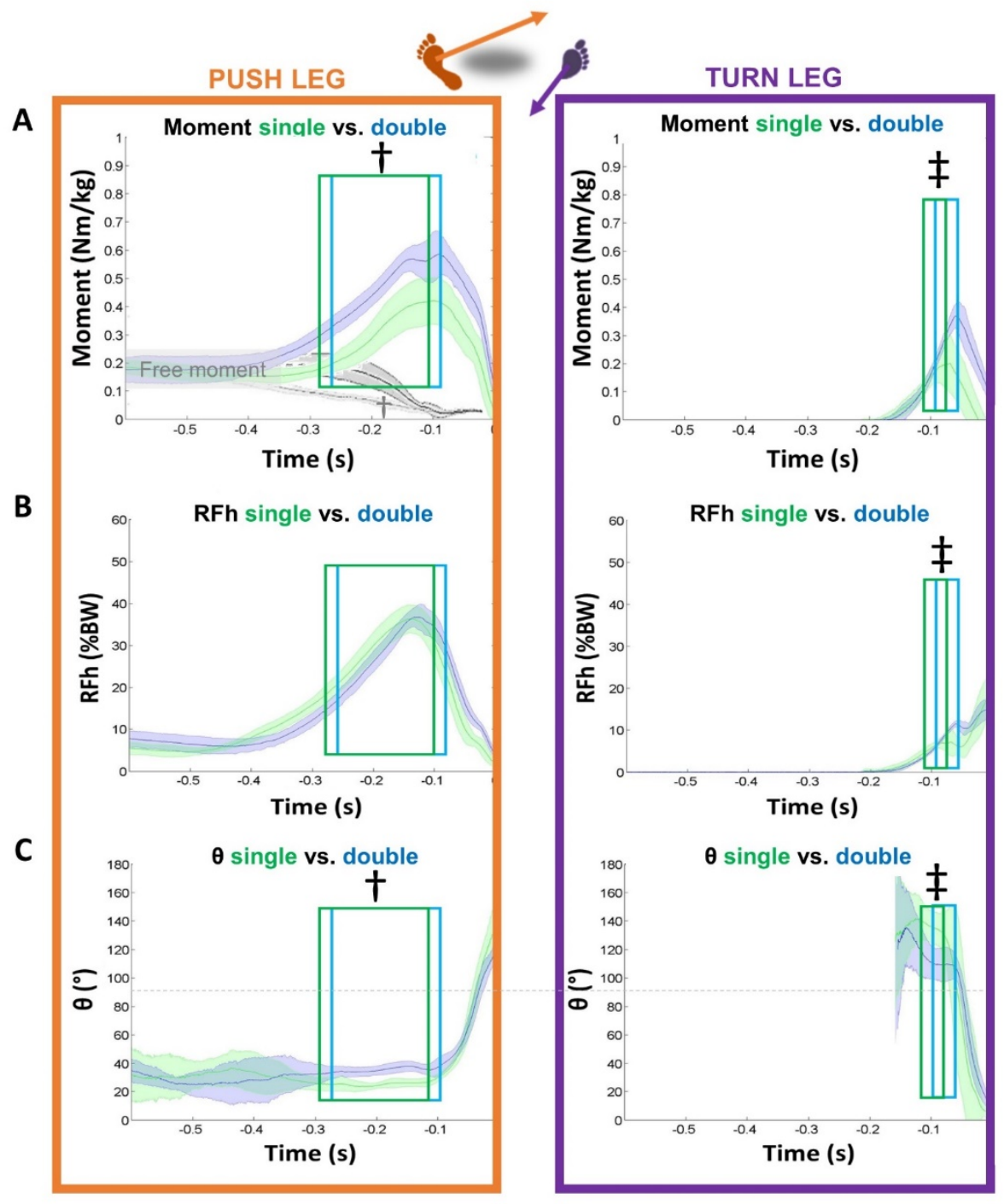

Figure 5: Control strategy used by an individual dancer (Subject 3) to increase net angular impulse generated during the initiations of single (light green) to double (darker blue) piqué turns. (A) Push leg and turn leg mean (SD) moment vs. time curves showing an increase in moment applied by each leg about a vertical line through the center of mass. Mean (SD) free moment superimposed in gray on the push leg's moment-time curve to show its large contribution to the total moment-time curve of the push leg. Light gray shows single piqué free moment, while darker gray shows larger free moment application during double piqués. (B) Increased mean (SD) magnitude of the horizontal reaction force (|RFh|) applied to the turn leg during rise to peak moment applied as rotational demand increased (C) horizontal reaction force redirection was used by both legs to increase mean (SD) $\sin \theta$ in doubles vs. singles. Boxed area displays $25 \%$ turn initiation phase duration prior to peak moment applied by each leg in each turn condition ("peak moment subphase"). Within-subject analysis revealed that with increased rotation, this dancer significantly increased her mean moment at both legs by increasing $\sin \theta$ at both legs $(\dagger, \ddagger)$ and the magnitude of RFh at the turn leg $(\ddagger)$. 


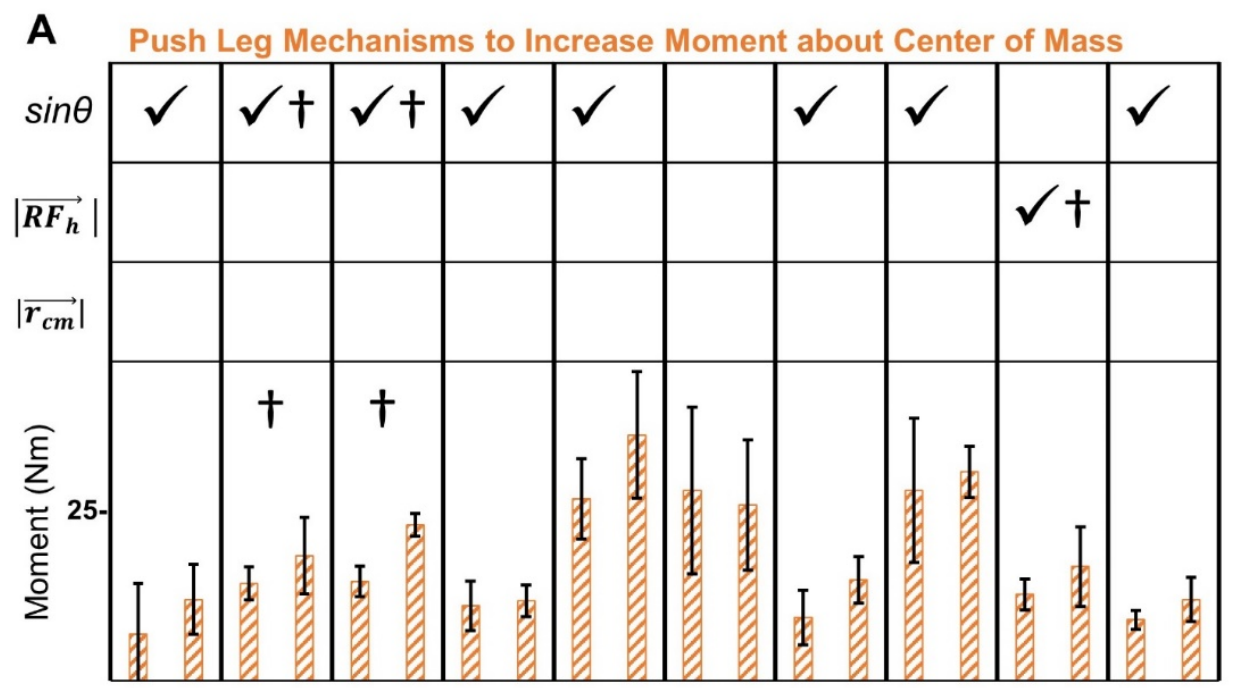

pk1 pk2 pk1 pk2 pk1 pk2 pk1 pk2 pk1 pk2 pk1 pk2 pk1 pk2 pk1 pk2 pk1 pk2 pk1 pk2 Sub1 Sub2 Sub3 Sub4 Sub5 Sub6 Sub7 Sub8 Sub9 Sub10

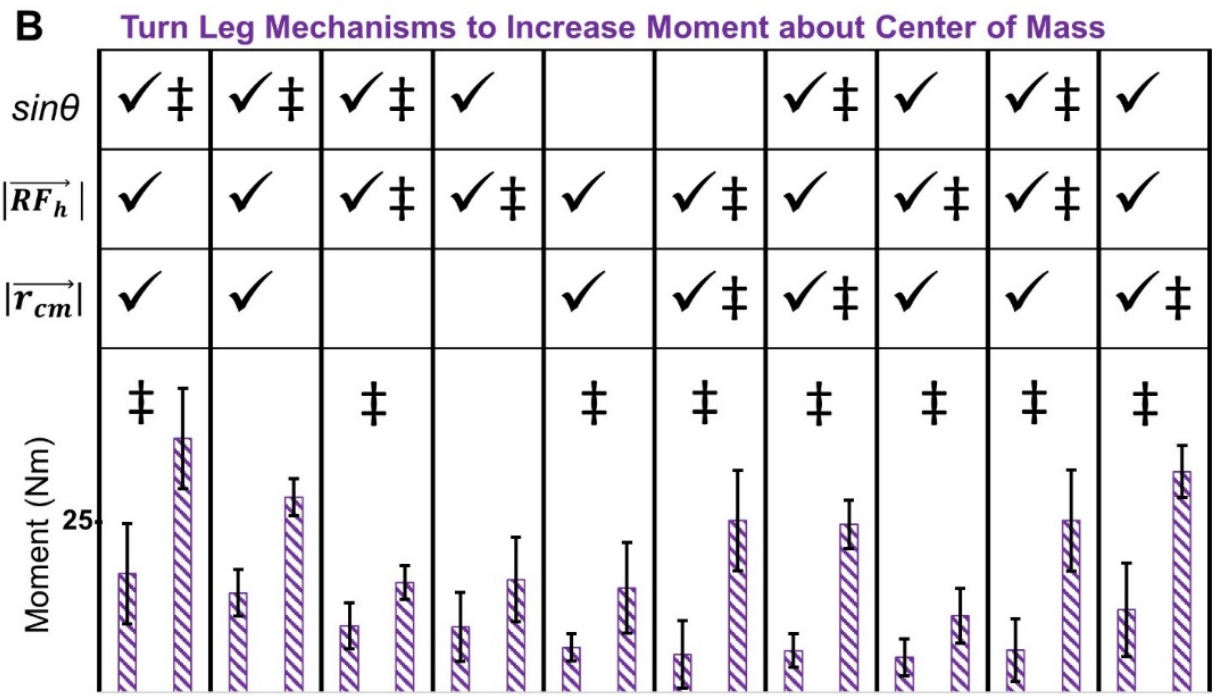

pk1 pk2 pk1 pk2 pk1 pk2 pk1 pk2 pk1 pk2 pk1 pk2 pk1 pk2 pk1 pk2 pk1 pk2 pk1 pk2 Sub1 Sub2 Sub3 Sub4 Sub5 Sub6 Sub7 Sub8 Sub9 Sub10

Figure 6: Subject-specific mechanisms used by dancers (Sub 1 - 11) to increase moment applied by the horizontal reaction force of the (A) push leg and (B) turn leg during the peak-moment subphase. Bargraphs indicate the mean (SD) average moment applied by the (A) push leg and (B) turn leg during the peak-moment subphase at each leg. If a subject increased a contributing factor to the moment applied by the horizontal reaction force $(\sin \theta$, horizontal reaction force magnitude $(|R F h|)$, and position vector length $(|\mathrm{rcm}|))$ with increased rotational demand during the peak-moment subphase is included in table form above each bargraph; where checkmarks $(\square)$ indicte a sustained increase in the variable's average value that exceeded its standard deviation and symbols indicate within-subject significant differences of the push leg $(\dagger)$ and turn leg $(\ddagger)$ variables between turn conditions (when tested at $\alpha=.05$ level and adjusted for multiple comparisons). + and $¥$ indicate within-subject significant differences between turn conditions when tested at $\alpha=.05$ level and adjusted for multiple comparisons. Group comparisons indicated increases in push leg $\sin \theta(p=.001)$, turn leg $|R F h|(p=.002)$, and turn leg $\sin \theta(p$ $=.002$ ) with increased rotation. 
Subject 2

Redirected reaction force
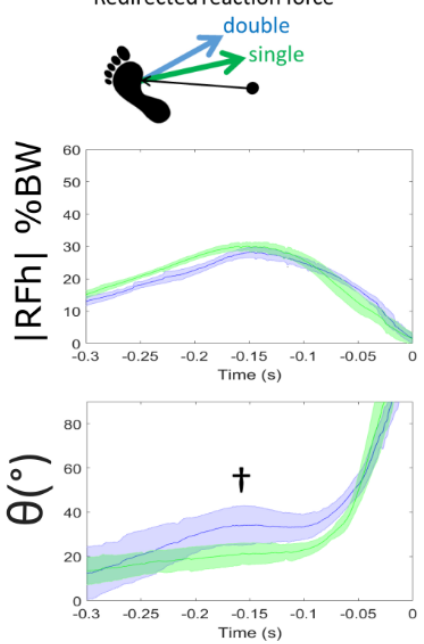

Subject 9

Increased reaction force
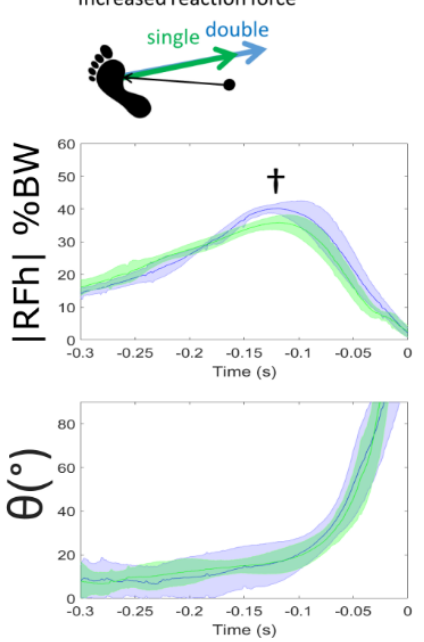

Figure 7: Example of contrasting subject-specific strategies used to increase the moment applied about the body's center of mass by the push leg during the second half of the initiation of a single turn (green) to a double turn (blue). Mean (SD) curves of the magnitude of the horizontal reaction force (|RFh|) and the angle between the RFh and position vector $(\theta)$ show that one dancer (Subject 2, left column) redirected the horizontal reaction force (increasing $\sin \theta$ ) without increasing horizontal reaction force magnitude, whereas, the other dancer (Subject 9, right column) used the opposite strategy. Both dancers successfully performed single and double turns without visual differences in kinematics and angular impulse generated by each dancer's push leg during single and double turns were within $5 \%$ of each other (body mass-normalized, Figure 2). However, these strategies are different from a musculoskeletal control perspective, which would merit different biofeedback training interventions to improve performance. 